\title{
Les réseaux inter-organisationnels comme espace de création de connaissance
}

\section{Alsones Balestrin et Pierre Fayard}

\section{(2) OpenEdition}

12 Journals

Édition électronique

URL : http://journals.openedition.org/communicationorganisation/2891

DOI : 10.4000/communicationorganisation.2891

ISSN : $1775-3546$

Éditeur

Presses universitaires de Bordeaux

Édition imprimée

Date de publication : 1 mai 2004

ISSN : 1168-5549

Référence électronique

Alsones Balestrin et Pierre Fayard, « Les réseaux inter-organisationnels comme espace de création de connaissance », Communication et organisation [En ligne], 24 | 2004, mis en ligne le 27 mars 2012, consulté le 19 avril 2019. URL : http://journals.openedition.org/communicationorganisation/2891 ; DOI : 10.4000/communicationorganisation.2891

Ce document a été généré automatiquement le 19 avril 2019

(c) Presses universitaires de Bordeaux 


\title{
Les réseaux inter-organisationnels comme espace de création de connaissance
}

\author{
Alsones Balestrin et Pierre Fayard
}

\section{Introduction}

1 La connaissance est reconnue comme un facteur clef de la compétitivité des organisations. Au-delà de sa gestion. Ikujiro Nonaka en prône la création comme un axe stratégique majeur. Les conditions favorables à celle-ci passent par la méthode SEC1 (Socialisation - Externalisation - Combinaison - Internalisation) mais surtout par l'émergence de $b a^{1}$ ou communautés stratégiques de connaissance. Par son origine, ce concept renvoie à la culture japonaise où l'approche du knowledge management diffère, notablement d'une nord-américaine, explicite et axée technologies de l'information.

2 Si les enjeux de la dite société de la connaissance s'imposent à l'échelle globale et qu'il en est de même pour ce qui est des potentiels et des orientations contenus dans les technologies de l'information et de la communication, les conditions mêmes de l'efficacité passent par une digestion culturelle locale, qui. en s'inspirant des autres modèles, crée une approche qui lui est propre et où ses valeurs comme ses atouts s'investissent de manière innovante. L'explicitation des cultures de la stratégie représente l'un des moyens de ce processus. Chacune d'entre elles définit le terreau à partir duquel germe la greffe de modèles de gestion et de création de connaissance opérationnelle pour les organisations.

Pour aborder la question de la génération de conditions favorables à la production de connaissance au sein d'une organisation, cette contribution propose d'en mesurer lu réalité à travers l'étude d'un réseau de PMI !, et de combiner à la fois réflexion théorique et résultats empiriques. Nous discuterons tout d'abord le concept de réseau horizontal dans sa relation avec la théorie de la création de connaissance opérationnelle, pour 
ensuite présenter les principaux résultats d'une étude de cas réalisée dans l'Etat brésilien du Rio Grande do Sul. Si des évidences empiriques semblent consacrer la pertinence de cette théorie à travers l'établissement d'un $b a$, il reste à solidifier et à prolonger la recherche par la considération et l'étude d'autres contextes culturels pour en asseoir la validité. En conclusion, cette-contribution discutera d'une méthode possible permettant, à partir de l'explicitation d'une culture de la stratégie, de définir des approches spécifiques de la question de la connaissance dans les organisations dans des cultures différentes.

\section{La création de connaissance opérationnelle}

4 La société informationnelle (Castells. 1999) pose comme facteur principal de productivité et de compétitivité la capacité à produire, à traiter et à transformer information et connaissance en actifs économiques. Nonaka distingue entre la connaissance tacite et la connaissance explicite exprimée quant à elle de manière formelle, systématique et partagée sous la forme de données, de formules scientifiques, de spécifications... En revanche, la connaissance tacite et individualisée n'est pas formalisée. L'épistémologie occidentale considère traditionnellement la dimension explicite de la connaissance là où Nonaka et al (2001) soulignent la complémentarité essentielle de ces deux dimensions au travers de la dynamique d'interactions interpersonnelles et avec leurs environnements pertinents respectifs. C'est pourquoi, lu connaissance créée s'enracine dans le système de valeurs des individus, de leurs contextes et de leurs cultures.

5 Ce processus de génération de connaissance opérationnelle au sein des organisations a été dénommé par Nonaka et al (2002) conversion de connaissance par laquelle tacite et explicite interfèrent et se transforment tant au niveau qualitatif que quantitatif, les quatre types de conversion reprennent les phases du modèle SECI. La socialisation convertit le tacite en tacite, l'extériorisation convertit le tacite en explicite, la combinaison convertit l'explicite en explicite et l'intériorisation ferme la boucle ou plutôt la spirale en convertissant l'explicite en tacite. Or un tel processus nécessite l'existence d'espaces et de temps appropriés que Nonaka et al. (2001) qualifie de ba, véritable plate-forme de création de connaissance. Ce concept de ba est susceptible d'unifier espace physique, virtuel et mental. 


\begin{tabular}{|c|c|c|c|}
\hline \multirow{2}{*}{\multicolumn{2}{|c|}{$\begin{array}{l}\text { Origining ba } \\
\text { Socialisation } \\
\text { Tacite }\end{array}$}} & \multicolumn{2}{|c|}{$\begin{array}{l}\text { Dialoguing ba } \\
\text { Extériorisation }\end{array}$} \\
\hline & & Tacite & Explicite \\
\hline \multicolumn{2}{|c|}{$\begin{array}{l}\text { Explicite } \\
\text { Intériorisation }\end{array}$} & Explicite & Explicite \\
\hline Exercisin & & Systemi & \\
\hline
\end{tabular}

Figure 01 : Le processus SECl et les types de ba. Adapté de Nonaka et al. 2002.

Originating ba est un espace d'empathie où les individus partagent émotions, sentiments, expériences, modèles mentaux... C'est là que s'initie le processus de création de connaissance associé au mécanisme de la socialisation où respect mutuel et confiance sont essentiels. Dialoguing ba permet la formalisation du tacite. À travers l'échange, il contribue à l'émergence collective d'une connaissance explicite à partir de l'extériorisation du tacite. Systemizing ba constitue le contexte favorable à la combinaison des nouvelles connaissances explicites avec celles qui leur préexistent. Les réseaux on-line et le groupware offrent un espace virtuel propice à cette conversion. Exercing ba incarne le contexte qui permet aux individus d'intérioriser le produit de la combinaison précédente en la transformant en connaissance facile comme par exemple un nouveau savoir-faire.

Dans chacun de ces ba sont créés des actifs de connaissance, soit des ressources spécifiques indispensables au processus de création de valeur pour l'organisation. La connaissance tacite partagée à travers des expériences communes (originating ba) est propice à l'émergence d'aptitudes, de savoir-faire, de confiance mutuelle, de dynamisme et de tension. La connaissance tacite explicitée et formalisée dans des symboles, des images et un langage commun (diaioguing ba) encourage la création d'actifs de connaissance comme des concepts de produits. La phase de systématisation (systemizing ba) enrichit les bases de données et la documentation en générale : spécifications, manuels, brevets, licences... Enfin, l'intériorisation à travers des actions et des nouvelles pratiques (Exercing ba) créent implémente savoir-faire, nouvelles capacités, normes de comportement organisationnel...

Dans une entreprise un ha peut être représenté par la structure organisationnelle intégrant les personnes et les processus de travail. Birkinshaw et al. (2002) ont observé qu'il existe des relations étroites entre connaissance et structure organisationnelle. Pour ces auteurs, la connaissance constitue une cinquième variable de contingence ${ }^{2}$ qui force l'adéquation de la structure organisationnelle. Ainsi, pour faciliter le processus de socialisation de connaissance tacite (généralement complexe et systémique) la structure organisationnelle devra faciliter les relations interpersonnelles et entre les personnes et leurs contextes. D'où la question de recherche: comment mettre les organisations en 
situation de production des conditions favorables à travers l'établissement d'un ha adéquat pour le processus de création de connaissance?

La justification de cette étude est fondée sur des évidences théoriques. Dyer et Nobeoka (2000) démontrent qu'un réseau organisationnel peut être-plus effectif qu'une entreprise intégrée dans la création, le partage cl la combinaison de connaissance. Nonaka et Nishiguchi (2001) soulignent l'importance de la coopération inter organisationnelle pour générer et partager de nouvelles connaissances. Castells (1999) propose deux critères de mesure de l'efficacité d'un réseau: la cohérence et la connexité. Nous faisons notre l'hypothèse selon laquelle les réseaux offrent les qualités nécessaires au processus de création de connaissance et reprenons l'incitation de Nonaka et Nishiguchi (2001:288): « we need to know more about how concepts such as ba and co-evolution can help small and medium-si/ ed firms developing régions".

\section{Le réseau horizontal comme espace de création de connaissance}

10 La création de nouvelles connaissances à partir du partage et du traitement d'information entre les organisations a été démontrée par Nonaka et al. (1997) par rapport à la dimension ontologique de la création de la connaissance. Celle dernière naît au stade individuel puis connaît une évolution qualitative susceptible de s'étendre jusqu'à l'intergénérationnelle. Selon ces auteurs, cette génération se manifeste lorsque la dynamique d'interaction tacite - explicite s'élève d'un niveau ontologique donné jusqu'à un niveau supérieur. On note ainsi que la connaissance n'est en définitive créée que par des individus mais que les organisations et les réseaux en fournissent les conditions favorables. En analysant la figure 2 ci dessous, on remarque que la connaissance inter organisationnelle créée dans le contexte d'un réseau d'entreprises, est l'une des dimensions les plus importantes de la création de la connaissance. Ce processus s'initie au niveau individuel puis, à travers l'interaction entre tacite et explicite, entre individus, groupes et organisations, il se transforme qualitativement de manière significative. Cela passe par un contexte favorable aux synergies et au partage d'émotions, d'expériences et d'images mentales. Un tel environnement ne saurait être construit sur le mode du command and control propre à un management pyramidal traditionnel mais plutôt sur le mode du energize and stimulate tel que le recommande Kikavvada (2000). 


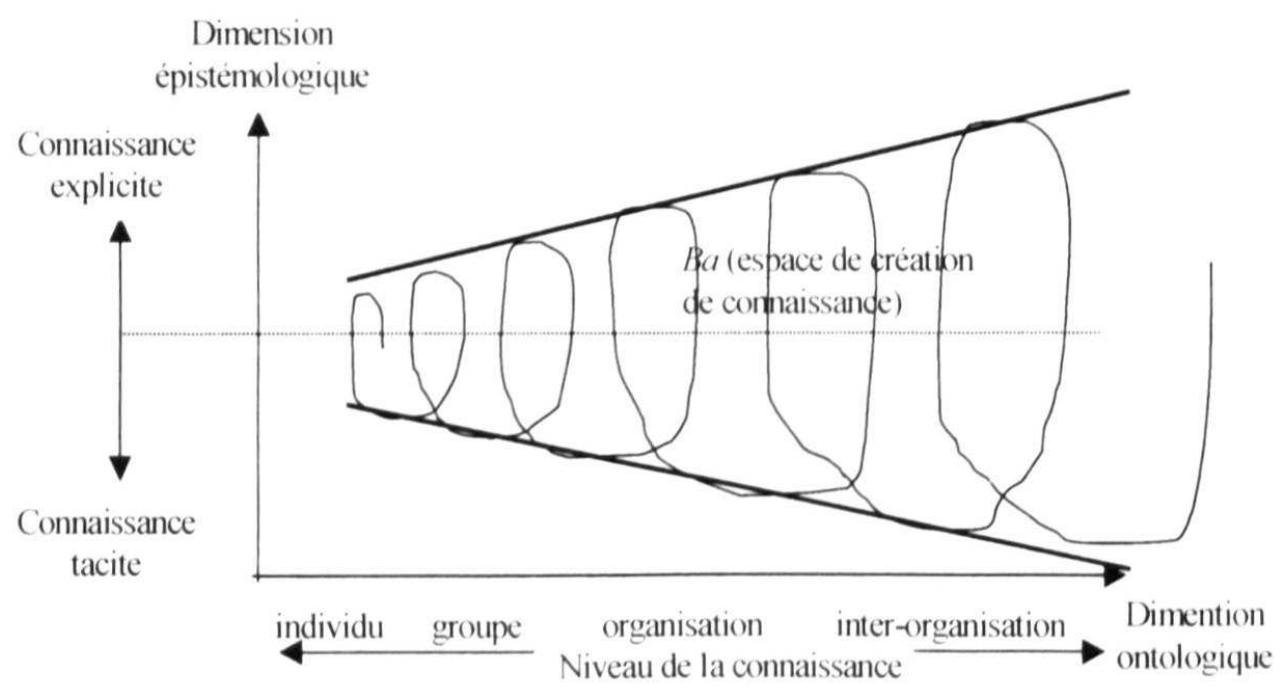

Figure 2. Spirale de création de lu connaissance.. Adaptée de Nonaka et al (1997)

\section{Le cas AGIVEST}

En décembre 2000. le gouvernement de l'Etat du Rio Grande do Sul au Brésil, a lancé le programme Réseaux de coopération pour stimuler par la performance des systèmes locaux de production au niveau mondial et favoriser la coopération entre PMEs. Deux années plus tard, trente trois réseaux de PMEs, positionnés sur des domaines d'activité divers, rassemblent un total de sept cent trente trois entreprises pour un total de plus de cinq mille salariés. Le projet compte avec la participation de six universités et quarante deux consultants contribuent à la formation et à la gestion de ces réseaux. Un ensemble de dispositions complémentaires facilite l'accès au crédit, développent la qualification des managers et aident à la participation aux salons professionnels ${ }^{3}$.

Nous avons choisi de rendre compte du réseau AGIVEST dont l'origine remonte à septembre 2001 et qui est constitué de quarante quatre PMEs du secteur textile. Les principales caractéristiques de ce réseau sont : a) la nature de TPEs ${ }^{4}$ des partenaires, soit une moyenne de six employés par entreprise, $b$ ) la proximité géographique dans un rayon de $100 \mathrm{~km}$ e) l'appartenance à un même secteur d'activité, d) la structure de gestion du réseau constituée par un président et un vice-président, supervisé par un conseil d'administration, un conseil d'éthique et pu- un conseiller fiscal, e) la prise de décisions stratégiques en assemblée générale et $\mathrm{f}$ ) le règlement de tout différent à travers l'application des instruments légaux: le statut du réseau, le code d'éthique et le régime interne. Ce réseau AGIVEST peut être qualifié de réseau horizontal de coopération en différenciation à d'autres typologies de réseaux de PMEs comme les réseaux verticaux ${ }^{5}$. Un réseau horizontal de coopération rassemble des entreprises qui ne peuvent atteindre certains objectifs stratégiques que collectivement. 


\section{La dynamique de création de connaissance au sein du réseau AGIVEST}

Cette recherche ${ }^{6}$ a permis d'observer le fonctionnement d'un réseau horizontal de TPEs par rapport à l'enjeu de la création de connaissance. Les évidences empiriques dégagées sont analysées à la lumière des réflexions théoriques précédentes. D'une manière générale, les managers reconnaissent unanimement que le plus grand bénéfice obtenu à travers la formation du réseau AGIVEST a été le partage d'information et de connaissance entre les entreprises participantes. Parmi celles-ci. les plus bénéfiques pour le réseau ont été celles concernant : les processus de production, les fournisseurs, les technologies et les marchés. Ces informations ont été partagées à travers une intensification d'interactions sociales souvent informelles. Plusieurs temps et espaces privilégiés ont été identifiés. Conformément aux orientations de Nonaka et al. (2002) cet ensemble de ha spécifiques constitue la plate-forme de création de connaissance du réseau.

\begin{tabular}{|c|c|}
\hline$B A$ & Evidences Empiriques \\
\hline $\begin{array}{l}\text { Réunion } \\
\text { dans les } \\
\text { entreprises }\end{array}$ & 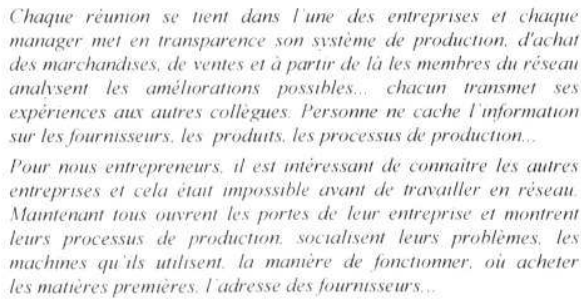 \\
\hline Assemblées & 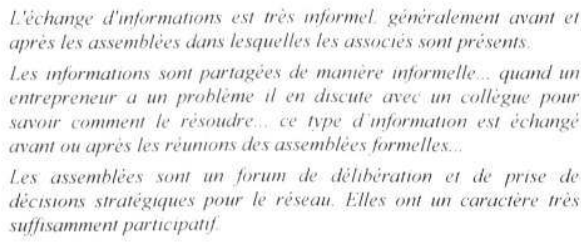 \\
\hline $\begin{array}{l}\text { Evénements } \\
\text { festifs }\end{array}$ & $\begin{array}{l}\text { Les repas. rendez-vous informel sont des moments intéressants de } \\
\text { partage de connaissance pour les entrepreneurs. les emplovés et les } \\
\text { proches impliqués dans le réseau. }\end{array}$ \\
\hline $\begin{array}{c}\text { Voyages et } \\
\text { visites de } \\
\text { salons }\end{array}$ & 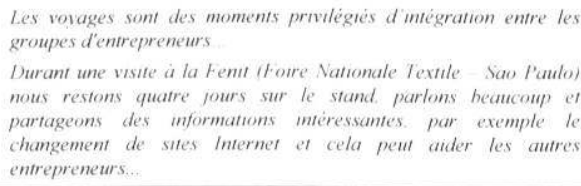 \\
\hline
\end{tabular}


Tableau 01 : Le processus de création de connaissance Evidence empirique de la recherche

\begin{tabular}{|c|c|}
\hline$B A$ & Evidences Empiriques \\
\hline \multirow[t]{2}{*}{$\begin{array}{l}\text { Sessions de } \\
\text { formations }\end{array}$} & $\begin{array}{l}\text { A partir du moment ou je suis entré dans le réseau j'ai commencé à } \\
\text { constater combien mon entreprise était désorganisée... je n'avais } \\
\text { pas le contróle des coùts. je ne savais pas diriger... je ne sais pas } \\
\text { comment jai pu survivre... à partir du moment où j ai commencé à } \\
\text { participer aux sessions de formation et à discuter avec les autres } \\
\text { collègues, jai découvert que nos problèmes sont très semblables et } \\
\text { que nous pouvons trouver les solutions ensemble. }\end{array}$ \\
\hline & $\begin{array}{l}\text { Nous avons suivi une session de } 120 \text { het cela a aussi permis un fort } \\
\text { échange d'information. }\end{array}$ \\
\hline $\begin{array}{l}\text { Planification } \\
\text { stratégique }\end{array}$ & $\begin{array}{l}\text { La planification stratégique est le moment où l'ensemble des } \\
\text { entrepreneurs élabore une vision commune sur les objectifs. les } \\
\text { défis et les opportunités du réseau pour l'avenir. }\end{array}$ \\
\hline $\begin{array}{l}\text { Espace } \\
\text { électronique }\end{array}$ & $\begin{array}{l}\text { Nous parlons beaucoup par téléphone. par courrier électronique et } \\
\text { échangeons des fax... }\end{array}$ \\
\hline
\end{tabular}

Tableau 01 : Continuation

14 Ces témoignages démontrent qu'il existe plusieurs ha (espaces physiques ou virtuels) en faveur du partage d'information et de connaissance. L'un de ceux-ci. surtout en termes de communication de connaissance tacite, est la visite des autres entreprises du réseau. À travers ces visites, les entrepreneurs identifient des possibilités d'améliorations de leurs process, de l'usage des technologies et de concepts de production à même de permette un plus de compétitivité. Avec l'évolution du réseau les relations sociales se fortifient et le niveau de coopération se renforce. L'assemblée constitue un espace important du partage de connaissance. Elle incarne un forum formel pour la prise collective de décisions stratégiques à l'intérieur d'une dynamique de débat et d'échanges, le jour de l'assemblée certains entrepreneurs arrivent en avance pour discuter de manière informelle avec les autres. À l'issue d'une assemblée, l'un des participants confie que les conversations informelles qui se tiennent avant ou après, concernent des sujets plus spécifiques: problèmes ou solutions dans la production, nouveau fournisseur ou représentant, une nouvelle matière première...

L'intensification des relations sociales et l'amitié dans les affaires vont de-paire avec un sens très fort de la communauté. Nous constatons ici la prégnance forte des valeurs de la culture brésilienne et plus particulièrement gauch ${ }^{7}$. Les événements festifs joue un rôle essentiel de solidification des relations de confiance entre les entrepreneurs, les employés et les proches. Les voyages, visites et participations aux salons professionnels fournissent l'occasion de connaître d'autres réalités et de réfléchir conjointement sur les opportunités, les tendances et les défis dans le monde de la mode, fors de la participation à une exposition des produits du réseau AGIVEST à l'occasion d'un des plus grands salons de la mode, la Fenit de Sào Paulo. les entrepreneurs ont observé que les produits les plus différenciés et les plus sophistiqués se sont le mieux vendus. Cette intelligence du marché 
pourra être mise à profit lors de l'élaboration des stratégies de produits pour les marchés des entreprises du réseau.

Pour améliorer les capacités en management des entrepreneurs, le Gouvernement de l'État de Rio Grande do Sul développent des cours de qualification. Des sessions de cent vingt heures permettent d'approfondissement des concepts et techniques de gestion d'entreprise. Cette question est extrêmement importante du fait que le réseau travaille avec une marque unique. L'ensemble des normes et processus de production doit être respecté par tous afin de garantir la qualité des produits de la marque AGIVEST, la planification est élaborée de forme participative par tous les entrepreneurs du réseau. Ainsi la réflexion collective, comme par exemple l'analyse de la matrice SWOT (Strenghts. Weaknesses, Opportunities e Threats). rend possible une vision à long terme de l'avenir du réseau, fa définition collective des objectifs, les stratégies à adopter, les chronogrammes... sont réalisés selon un processus d'apprentissage pour l'ensemble des entreprises, les TIC sont encore insuffisamment utilisées au profit du fax et du téléphone et cela fragilise la dynamique de création de connaissance du réseau.

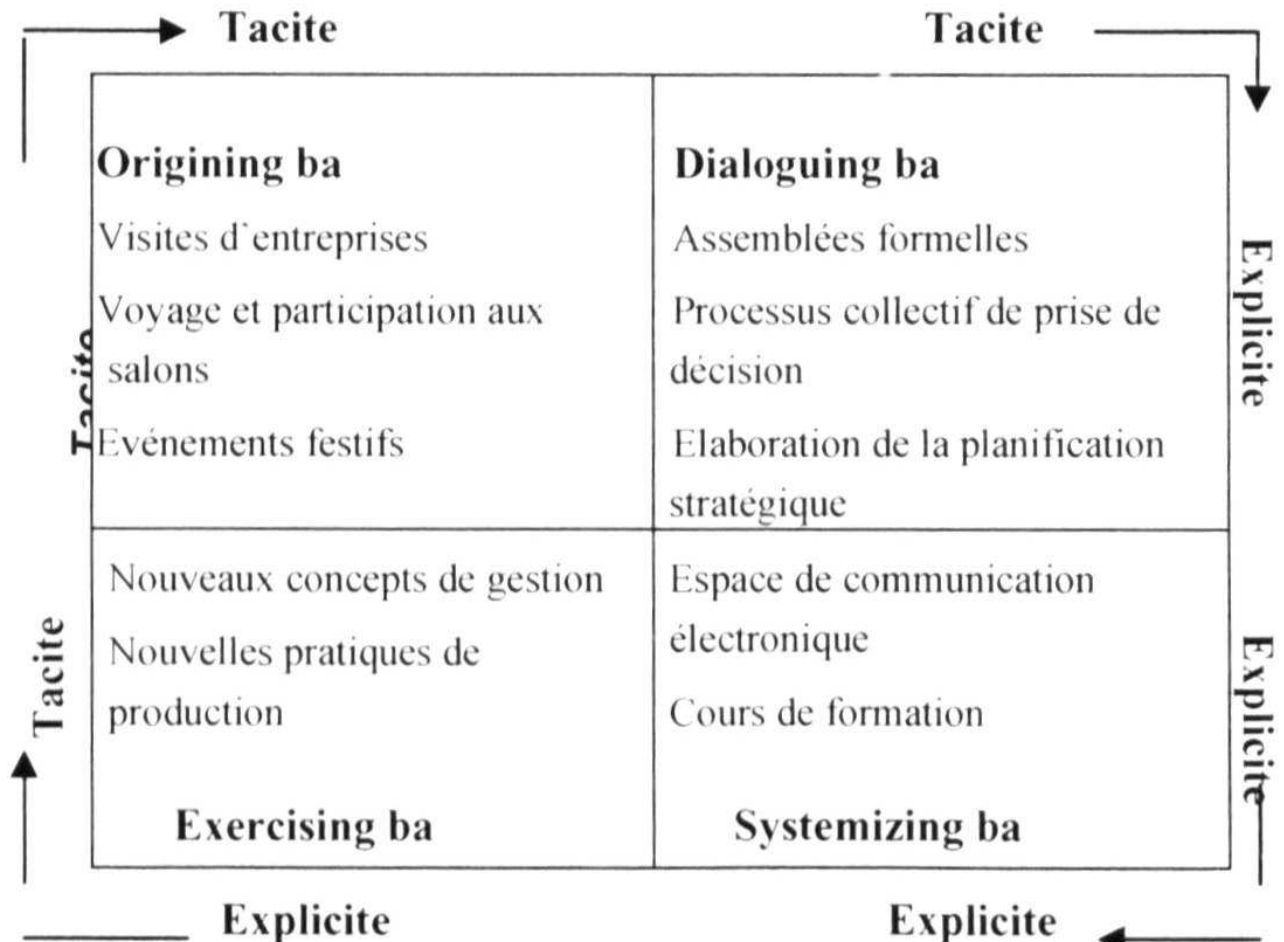

17 Origining ba est l'espace dévolu à la socialisation de la connaissance tacite. Cette phase est de plus en plus importante dans le processus de création de connaissance dans la mesure où les personnes perçoivent la connaissance tacite des autres. Au réseau AGIVEST origining ba résulte de différents temps et moments comme les visites au cours desquelles les entrepreneurs observent directement les solutions et les meilleures pratiques déjà adoptées par d'autres. Conformément aux réflexions de Nonaka \& Nishiguchi (2001). nous avons observé que dans l'AGIVEST origining ba est un espace d'interaction face-à-face favorable à la confiance et au compromis. Dialoguing ba se manifeste lors des assemblées formelles, des réunions d'élaboration de la planification stratégique et des autres processus collectifs de prises de décisions. Ces activités servent de plate-forme pour les entrepreneurs, au moyen du dialogue et de la réflexion collective, pour le partage et 
conversion de leur connaissance tacite en des termes et concepts communs (connaissance explicite - des modèles, des hypothèses et des scénarios du futur).

Systemizing ha est le contexte qui permet aux entrepreneurs combiner les nouvelles connaissances explicites à d'autres disponibles et préexistantes. C'est ici que les TIC pourraient jouer un rôle majeur en mettant à disposition de manière rapide et précise un capital cognitif. Dans le réseau AGIVEST. cette phase présente des insuffisances: inexistence d'un système intégré qui permettrait la gestion électronique des processus d'achat, d'approvisionnements, d'organisation de la production, de la gestion des clients... fors de la dernière phase du processus de création de la connaissance, exercising ba, la connaissance est intériorise et appliquée au travers des nouvelles pratiques organisationnelles. Pour Nonaka (1997) lorsque les individus l'intériorisent sous la forme de modèles mentaux ou de know-how techniques, les expériences personnelles peuvent devenir des actifs précieux pour l'organisation.

Des processus de création de connaissance opérationnelle, supportés par les divers types de ba. rendent possible l'émergence d'actifs de connaissance essentiels pour la création de valeur et d'avantages compétitifs pour les entreprises. C'est ce que l'on observe dans le cas du réseau AGIVEST seulement après un an d'existence. Ces actifs de connaissance apportent des avantages concurrentiels par rapport à des PMEs qui travaillent de façon individuelle. 1) Nouveaux concepts et savoir-faire de production : après être entré dans le réseau mon entreprise a amélioré les processus de production. en changeant seulement quelques processus, nous les avons amélioré de $40 \%$ sans augmenter ni le personnel ni les machines. 2) Nouveaux concepts de-produits et de marchés: les sociétés qui ont participé à la foire nationale du textile, ont observé que les acheteurs préféraient les produits différenciés et plus sophistiqués aux produits traditionnels, même lorsqu' 'ils sont plus chers. 3) Synergie et coopération : à partir du moment où je suis entré dans le réseau. AGIVEST j'ai commencé il participer à des sessions de formation et à discuter avec mes autres collègues... j'ai découvert que les problèmes sont très semblables et que les solutions peuvent être trouvées ensemble. D'autres actifs de connaissance produits par le réseau englobent les nouveaux designs de produits, les concepts communs pour une meilleure compréhension des perspectives de marchés, les brevets de la marque, les spécifications de-produits, les connaissances sur les fournisseurs et les représentants ou encore celles sur de nouvelles technologies et matières premières.

Cette élude de cas a eu comme principale finalité la compréhension de la dynamique de création de connaissance dans un réseau de TPEs. La perspective théorique de l'analyse se focalise sur le contexte et l'espace dans lequel la connaissance est créée, partagée et utilisée soit à travers le principe du ha ou communautés stratégiques de connaissance (Fayard. 2003). L'existence de ce type de communauté se rattache directement à des structures organisationnelles qui agencent personnes, valeurs et processus de travail. Les réseaux horizontaux constituent une configuration favorable à l'existence d'un ha approprié. Il reste à poursuivre et à approfondir la question stratégique du comment structurer (redisigner) les organisations pour qu'elles aménagent les conditions favorables à la création de connaissance. Pour ce faire, la prise en compte de la dimension culturelle, et notamment de la place sociale des dimensions et suprématies éventuelles du tacite et de l'explicite est essentielle. 


\section{BIBLIOGRAPHIE}

BIRKINSHAW. J.. NOBEL. R.. RIDDERSTRALE. J., Knowledge as a contingency variable: do the characteristics of knowledge predici organization structure? Organization Science. Max -June 2002. T.13. n.3.

BHAGAT. R. S.: KEDIA. B. I.: HARVESTON. P. D.. TRIANDIS. H.C.. Cultural variations in the crossborder transfer of organizational knowledge: an integrative framework. Academy of Management Review, 2002. 1.27. n.2. p. 204-221.

CASTELLS. M...1 era da informação : economia, sociedade e cultura. São Paulo : Paz e Terra. 1999.

DYER..J. H.. NOBEOKA. K.. Creating and managing a high-performance knowledge-charing network : the Toyota case. Strategie Management Journal. 2000. t. 21. p. 345-367.

DONALDSON. I... Teoria da contingencia estrutural. in : CLEGG. S.. HARDY. C. NORD. W.. Handbook de estudos organizacionais São Paulo : Atlas. 1999.

FAYARD. P.. La maitrise de l'interaction. L'information et la communication dans la stratégie. Paris : Zéro Heure Editions Culturelles. 2000.

FAYARI). P.. Culture de la stratégie el technologies de I 'interaction. La voie japonaise dans la création de la connaissance. Rapport Ambassade de France au Japon, avril 2002. consultable sur wwvv.adit.fr. FAYARD. P.. le concept de «ba » dans la voie japonaise de la création de la connaissance. Rapport Ambassade de F'ranco au Japon, juin 2003. consultable sur www.adit.fr.

HUMAN. S.F., PROVAN. K.Ci.. An emergent theory of structure and outcomes in small-firm strategic manufacturing network. Academy of Management Journal. 1997. t. 40. n.2. p. 368-403.

INZERLL. G. The Italian alternative: flexible organization ami social management. International Studies of Management \& Organization. 1990. t. 20. p. 6-21.

KIKAWADA. K.. Knowledge Dynamic Initiative. Fuji Xerox. Tokyo. 2000.

LORENZONI. G.. ORNATI. O.. Constellations of linns and new ventures. Journal of Business Venturing. 19X8. t. 3.p. 41-57.

NOHRIA. N.. FCCI.FS. R.. Networks and organizations: structure, form and action. Boston: Harvard Business School Press. 1992.

NONAKA. I: TAKEUCHI. I H. Criação de conhecimento na empresa. Rio de Janeiro : Campus. 1997.

NONAKA. I.. NISHIGUCHI. T.. Knowledge Emergence. New York: Oxford. 2001.

NONAKA. [.. TOYAMA. R... KONNO. N.. SECI. ha and leadership: a unified model of dynamic knowledge creation, in: Managing Knowledge an essential reader. London: Sage Publications. 2002.

OLIVER. A.L... EBERS. M.. Networking network studies: an analysis of conceptual configurations in the study of inter-organizational relationships. Organization Studies. 1998.1.19. p. 549-583.

PERROW. C. Small-firm Networks, in: NOIIRIA. N.: I (CI. IS. R. Networks and organizations: structure, form and action. Boston: Harvard Business School Press. 1992.

SOO. C; DEVINNEY. T: MIDGLEY. A.. Knowledge Management: philosophy, processes and pitfalls. California Management Review. Summer 2002.1.44. n.4. 


\section{NOTES}

1. Idéogramme japonais signifiant espace physique, virtuel ou mental favorable a la création de connaissance Voir rapports d'ambassade février 2002 \& juin 2003. www.adil fr.

2. Les autres variables étant la complexité environnementale, la stratégie, la technologie cl la taille de l'organisation (Donaldson. 1999).

3. Source AGIVEST. En août 2002

4. 1res Petite Entreprise

5. Typologie de réseau dans lequel le PMEs fournissent des services et des produits pour autres entreprises, généralement grands

6. La recherche a été réalisée grâce à la collecte de données primaires à travers cinq entretiens menés par Alsones Balestrin avec des entrepreneurs du réseau, avec le consultant du réseau et avec son président

7. Soit de l'Etat de Rio Grande do Sul tres entreprenant

\section{AUTEURS}

\section{ALSONES BALESTRIN}

Université de Poitiers. Université Fédérale du Rio Grande do Sul. Université UNISINOS/

Brésil

\section{PIERRE FAYARD}

Université de Poitiers ICOMMTEC 